INRA Prod. Anim., $1995,8(4), 273-274$

\section{J. ANDRIEU}

INRA Station de Recherches sur la Nutrition des Herbivores Theix 63122 St-Genès-Champanelle

\title{
Prévision de la digestibilité et de la valeur énergétique du maïs fourrage à l'état frais
}

La digestibilité de la matière organique (DMO) du maïs plante entière en vert et, par là, sa valeur énergétique, est sans doute moins variable que celle des autres fourrages mais peut cependant varier de façon non négligeable. C'est ainsi qu'elle a été en moyenne de $71,7 \%$ et a varié de 67 à $78 \%$ pour les 226 échantillons (autres que ceux portant le gène bm3) étudiés par le «Club Digestibilité " en 1987 et 1988 (Andrieu et al 1993). Ces variations sont dues au stade de maturité à la récolte, à la variété, à l'année et au lieu de culture. Les différences éventuelles entre variétés, pour un lieu et une année donnés, ne se retrouvent pas forcément cette même année dans un autre lieu, ni l'année suivante dans le même lieu. Il peut donc être intéressant de pouvoir prévoir au laboratoire la digestibilité du maïs en vert tout en sachant que les valeurs obtenues sont directement transposables au même maïs après ensilage. En effet, les différences de valeur UF entre le maïs en vert et le maïs ensilé sont en théorie inférieures à 0,01 UFL ou UFV et peuvent donc être négligées.

Suite aux travaux du "Club Digestibilité ", un document intitulé "Mieux calculer la valeur énergétique du maïs ensilage " avait été largement diffusé en 1991. Il y figurait 4 équations permettant de prévoir la DMO du maïs plante entière. L'application de ces équations posait cependant des problèmes essentiels :

Tableau 1. Méthodes de références.

(1) Les UF ont été calculées selon la démarche analytique proposée dans le bulletin du BIPEA n ${ }^{0} 244$.

\begin{tabular}{|l|l|l|}
\hline Cendres & MM & incinération 550 ${ }^{\circ} \mathrm{C}$ \\
MAT & MAT & Kjeldahl $(\mathrm{N} \times 6,25)$ \\
Amidon & AMI & Ewers (1910) \\
Cellulose brute & CB & Weende \\
NDF & NDF & Méthode Van Søest (1967): \\
ADF & ADF & - adaptée par Giger (1979) \\
ADL & ADL & - avec correction cendres \\
& & seulement sur ADL \\
Digestibilité & & \\
enzymatique (MS \%) & DCS & Méthode 0,1 N Aufrère(1983) \\
Digestibilité in vivo & DMO & 6 moutons en quantité \\
& & limitée (niveau \\
& & d'alimentation $=1,2)$ \\
UFL, UFV & & Systèmes INRA 1988 \\
\hline
\end{tabular}

- les résultats du Club avaient mis en évidence des différences importantes entre années ;

- la DMO prévue était systématiquement un peu différente suivant que l'équation retenue faisait uniquement appel à des critères chimiques ou comprenait aussi la digestibilité cellulasique (DCS) selon la méthode de Aufrère et al (1983). Cela résultait du fait que la DCS avait été déterminée par un laboratoire qui n'utilisait pas les mêmes creusets filtrants que ceux ayant servi à mettre au point la méthode à l'INRA de Theix. Les valeurs de DCS étaient systématiquement un peu supérieures à celles obtenues à l'INRA de Theix et l'équation faisant intervenir la DCS entraînait une sous-estimation de la DMO par les laboratoires d'analyses qui s'étaient «calés", pour la détermination de la DCS, sur les valeurs obtenues à Theix.

- enfin, une fois la DMO prévue, toute une série de calculs était nécessaire pour obtenir la valeur énergétique en UFL et UFV.

La remise à jour des équations que nous proposons ici a donc 3 objectifs principaux :

- rendre plus robustes les équations vis-àvis de l'effet année en intégrant 34 nouvelles mesures obtenues à l'INRA de Theix en 1992, 1993 et 1994.

- rétablir la cohérence entre la méthode chimique et la méthode enzymatique de J. Aufrère. A cette fin nous avons repris à Theix les mesures de digestibilité à la pepsine cellulase sur l'ensemble des échantillons du «Club Digestibilité » et de l'INRA pour les intégrer dans les nouvelles équations.

- simplifier la prévision des valeurs UF en proposant des équations directes à partir de la composition et de la digestibilité enzymatique.

\section{Nouvelles équations}

Au total 254 échantillons ont été utilisés dont 220 sont issus du "Club Digestibilité ". Parmi ces derniers, les maïs bm3 ont été éliminés. Les méthodes de références ainsi que les abréviations des variables sont données dans le tableau 1. Pour chacune des variables, la valeur moyenne, la plage de variation et l'écart-type sont donnés dans le tableau 2. 
Tableau 2. Valeurs moyennes, plages de variation et écarts-types (ET) des 254 échantillons. La composition chimique est exprimée en $\mathrm{g} / \mathrm{kg}$ de MO (lettre $\varnothing$ en indice), la digestibilité de la $M O$ et la digestiblité enzymatique de la MS en $\%$, les valeurs UF par $100 \mathrm{~kg}$ de $\mathrm{MO}$.

Tableau 3. Prévision de la digestibilité de la matière organique (DMO en \%) et des valeurs UFL et UFV (par $100 \mathrm{~kg}$ de MO) à partir de la composition chimique (en $\mathrm{g} / \mathrm{kg}$ de $\mathrm{MO}$ ) et de la digestibilité enzymatique de la MS (DCS en \%).

\begin{tabular}{|l|c|c|c|c|}
\hline Constituant & Moyenne & Min & Max & ET \\
\hline MAT $_{\varnothing}$ & 79 & 53 & 106 & 10 \\
AMI $_{\varnothing}$ & 254 & 42 & 422 & 81 \\
CB $_{\varnothing}$ & 219 & 163 & 300 & 24 \\
NDF $_{\varnothing}$ & 494 & 403 & 616 & 38 \\
$\mathrm{ADF}_{\varnothing}$ & 241 & 180 & 324 & 27 \\
$\mathrm{ADL}_{\varnothing}$ & 26 & 17 & 40 & 4 \\
$\mathrm{DCS}$ & 67,9 & 59,0 & 74,7 & 2,8 \\
$\mathrm{DMO}$ & 71,6 & 66,1 & 77,6 & 2,6 \\
$\mathrm{UFL}_{\varnothing}$ & 94,3 & 84,2 & 105,9 & 4,6 \\
UFV $_{\varnothing}$ & 83,9 & 72,5 & 96,9 & 5,2 \\
\hline
\end{tabular}

Les valeurs minimum et maximum des variables explicatives constituent les bornes d'utilisation des équations. La teneur en matière sèche des 254 échantillons a été en moyenne de $29 \%$ et a varié de 18 à $53 \%$.

Les variables expliquées (UF) et explicatives (composition chimique) ont été exprimées par rapport à la matière organique (lettre $\varnothing$ en indice). Les valeurs UFV ont été corrigées pour les interactions digestives conformément aux dispositions prises lors de la réactualisation des systèmes INRA en
1988. Quatre modèles ont été testés avec les variables suivantes :

$$
\begin{array}{llll}
\text { M1 } & \text { MAT }_{\varnothing}, & \mathrm{CB}_{\varnothing}, & \mathrm{AMI}_{\varnothing} \\
\text { M2 } & \text { MAT }_{\varnothing}, & \mathrm{NDF}_{\varnothing}, & \mathrm{ADF}_{\varnothing}, \mathrm{ADL}_{\varnothing} \\
\text { M3 } & \text { MAT }_{\varnothing}, & \mathrm{AMI}_{\varnothing}, & \mathrm{DCS} \\
\text { M4 } & \text { MAT }_{\varnothing}, & \text { DCS }
\end{array}
$$

Les variables explicatives proposées n'ont été retenues dans le modèle que si elles étaient significatives à $\mathrm{P}=0,05$ (tableau 3 ). Un exemple d'application est donné au tableau 4.

\section{Conclusion}

Les nouvelles équations permettent d'obtenir facilement des valeurs UF comparables quel que soit le modèle. Elles devraient être plus robustes vis-à-vis de l'effet année même si leurs écart-types résiduels sont peu modifiés par rapport aux équations du «Club Digestibilité ». Comme dans le cas des résultats du Club, nous avons observé que la spec-

\begin{tabular}{|c|c|c|c|c|c|c|c|c|c|c|c|}
\hline & & & & & & & & & & $\mathrm{R}$ & RSD \\
\hline M1 & DMO & $=$ & 79,4 & + & $0,0652 \mathrm{MAT}_{\varnothing}$ & - & $0,0591 \mathrm{CB}_{\varnothing}$ & & & 0,634 & 1,98 \\
\hline M2 & DMO & $=$ & 75,7 & + & $0,0701 \mathrm{MAT}_{\varnothing}$ & + & $0,0156 \mathrm{NDF}_{\varnothing}$ & - & $0,0720 \mathrm{ADF}_{\emptyset}$ & 0,643 & 1,97 \\
\hline M3* & DMO & $=$ & 32,5 & + & $0,0764 \mathrm{MAT}_{\varnothing}$ & + & $0,0049 \mathrm{AMI}_{\varnothing}$ & + & $0,4700 \mathrm{DCS}$ & 0,680 & 1,88 \\
\hline M4 & DMO & $=$ & 28,5 & + & $0,0732 \mathrm{MAT}_{\varnothing}$ & + & $0,5501 \mathrm{DCS}$ & & & 0,668 & 1,91 \\
\hline M1 & $\mathrm{UFL}_{\varnothing}$ & $=$ & 110,15 & + & $0,1233 \mathrm{MAT}_{\varnothing}$ & - & $0,1167 \mathrm{CB}_{\oslash}$ & & & 0,683 & 3,40 \\
\hline M2 & $\mathrm{UFL}_{\varnothing}$ & $=$ & 103,95 & + & $0,1339 \mathrm{MAT}_{\varnothing}$ & + & $0,0255 \mathrm{NDF}_{\square}$ & - & $0,1358 \mathrm{ADF}_{\emptyset}$ & 0,691 & 3,37 \\
\hline M3* & $\mathrm{UFL}_{\emptyset}$ & $=$ & 20,20 & + & $0,1462 \mathrm{MAT}_{\varnothing}$ & + & $0,0109 \mathrm{AMI}_{\varnothing}$ & + & $0,8818 \mathrm{DCS}$ & 0,724 & 3,22 \\
\hline M4 & $\mathrm{UFL}_{\mathscr{D}}$ & $=$ & 11,38 & + & $0,1390 \mathrm{MAT}_{\varnothing}$ & + & 1,0609 DCS & & & 0,707 & 3,29 \\
\hline M1 & $\mathrm{UFV}_{\varnothing}$ & $=$ & 102,49 & + & $0,1297 \mathrm{MAT}_{\varnothing}$ & - & $0,1318 \mathrm{CB}_{\varnothing}$ & & & 0,678 & 3,84 \\
\hline $\mathrm{M} 2$ & $\mathrm{UFV}_{\varnothing}$ & $=$ & 95,50 & + & $0,1416 \mathrm{MAT}_{\varnothing}$ & + & $0,0287 \mathrm{NDF}_{8}$ & - & $0,1534 \mathrm{ADF}_{\varnothing}$ & 0,687 & 3,81 \\
\hline M3* & $\mathrm{UFV}_{\varnothing}$ & $=$ & 0,76 & + & $0,1555 \mathrm{MAT}_{\varnothing}$ & + & $0,0123 \mathrm{AMI}_{\varnothing}$ & + & $0,9984 \mathrm{DCS}$ & 0,720 & 3,64 \\
\hline M4 & $\mathrm{UFV}_{\varnothing}$ & $=$ & $-9,12$ & + & $0,1475 \mathrm{MAT}_{\varnothing}$ & + & $1,1992 \mathrm{DCS}$ & & & 0,703 & 3,72 \\
\hline
\end{tabular}
trophotométrie dans le proche Infra-rouge permettait d'améliorer la précision par rapport à celle des modèles proposés plus haut.

* L'amélioration de la précision liée à la prise en compte de la teneur en amidon (comparaison entre M3 et M4) étant faible, on pourra s'affranchir de sa détermination et utiliser le modèle M4.

Tableau 4. Exemple d'application à partir des résultats du circuit fourrage $n^{\circ} 261$ du BIPEA (Bureau Interprofessionnel d'Etudes Analytiques).

\begin{tabular}{|l|c|c|c|c|c|c|c|c|}
\hline \multicolumn{4}{|l|}{$\begin{array}{l}\text { Composition } \\
\text { (Références circuit) }\end{array}$} & $\begin{array}{l}\text { Valeurs } \\
\text { “vraies " }\end{array}$ & \multicolumn{3}{|c|}{ Estimation à partir des modèles } \\
\hline & g/kg MS & g/kgMO & Y & $(1)$ & M1 & M2 & M3 & M4 \\
\hline MM & 50,1 & $/$ & DMO & 73,7 & 74,4 & 73,7 & 73,5 & 73,2 \\
MO & 949,9 & $/$ & & & & & & \\
MAT & 83,4 & 88 & UFL $_{\varnothing}$ & 98,2 & 99,8 & 98,4 & 98,0 & 97,3 \\
AMI & 320,3 & 337 & UFV $_{\varnothing}$ & 88,2 & 98,9 & 88,4 & 88,0 & 87,2 \\
CB & 172,8 & 182 & & & & & & \\
NDF & 463,6 & 488 & UFLS $^{(3)}$ & 93,1 & 94,8 & 93,5 & 93,1 & 92,5 \\
ADF & 208,2 & 219 & UFVS $^{\prime}$ & 83,6 & 85,4 & 83,9 & 83,6 & 82,8 \\
ADL & 26,2 & 28 & & & & & & \\
DCS & $69,5(2)$ & $/$ & & & & & & \\
\hline
\end{tabular}

(1) Valeurs mesurées (DMO) ou calculées (UF) selon les démarches proposés dans le bulletin BIPEA $\mathrm{n}^{0} 244$. Il est à noter que la composition chimique, la digestibilité et le niveau d'alimentation retenus dans ce calcul sont ceux de l'essai INRA.

(2) $\operatorname{En} \%$.

(3) Pour $100 \mathrm{~kg}$ de MS.

\section{Abstract}

254 in vivo trials on sheep led to prediction models for in vivo organic matter digestibility (DMO) and net energy value of green whole plant maize used for silage. The proposed equations are able to predict DMO with a residual standard deviation from 1.88 to 1.98 point according to used predictors (chemical constituents and (a) pepsin-cellulase digestibility).

\section{Références bibliographiques}

Andrieu J., Demarquilly C., Dardenne P., Barrière Y., Lila M., Maupetit P., Rivière F., Femenias N., 1993. Composition and nutritive value of whole maize plants fed fresch to sheep. I. Factors of variation. Ann. Zootech., 42, 221-249.

Aufrère J., Michalet-Doreau B., 1983. In vivo digestibility and prediction of digestibility of some by-products. EEC Seminar, 26-29 September 1983, Mlle Gontrode, Belgique. 